\title{
Edulcorantes no nutritivos, riesgos, apetito y ganancia de peso
}

\author{
Non-nutritive sweeteners risks, \\ appetite and weight gain
}

\begin{abstract}
Artificial sweeteners are substances that provide energy and are added to foods to provide sweetness, increasing the pleasure of eating. The aim of the following review is to report on the current literature regarding artificial sweeteners, risks related to consumption, and possible effects on appetite and weight gain. Since the introduction of the artificial sweeteners, the media have reported the potential risks of cancer, which has undermined the sense of public safety. The role of sweeteners on cancer risk has been widely debated in recent decades. Recent studies indicate no adverse effects on the consumption of saccharin, aspartame, acesulfame $k$ and other sweeteners. This includes data on cancers of the stomach, pancreas and endometrium. Today many artificial sweeteners are combined in current products, the carcinogenic risk of a single substance is difficult to assess. Consumption of sweet flavors in the absence of calories produced significantly different effects compared to the consumption of sweet flavors associated with calories, and over time these effects may contribute to a positive energy balance and increased body weight gain . Key words: Non-nutritive sweeteners, appetite, cancer.
\end{abstract}

\section{INTRODUCCIÓN}

Desde tiempos ancestrales la humanidad ha tenido una marcada preferencia hacia los alimentos dulces (1). A fines del siglo XVII se decía que el azúcar era responsable de provocar un gran número de enfermedades y surgió la necesidad de buscar un aditivo que pudiera sustituir el azúcar de los alimentos, sumado a que durante la Segunda Guerra Mundial hubo una escasez importante de azúcar y un cambio de la estética a favor de una figura delgada animó a las mujeres a recurrir a sustitutos artificiales (2).

Los edulcorantes debían proporcionar las mismas cualidades y sensaciones que producía el azúcar en los alimentos (1).

Los edulcorantes artificiales han ganado espacio como herramientas de la dieta (3) ya que proporcionan el sabor dulce del azúcar, pero sin el aporte calórico de esta, por lo tanto pueden ayudar a bajar de peso y a la adhesión de la dieta. Los edulcorantes artificiales se emplean para reemplazar total o parcialmente el azúcar, además poseen un mayor poder endulzante que la sacarosa (30 a 300 veces) y son más económicos. Pueden contribuir al control de peso o de glucosa en sangre (4).

Edulcorantes utilizados en la industria alimentaria Los edulcorantes utilizados en la industria alimentaria
Samuel Durán A. (1)

Karla Cordón A. (2)

María del Pilar Rodríguez N. (3)

(1) Facultad de Ciencias de la Salud, Universidad San Sebastián, Santiago, Chile. (2) Universidad de San Carlos de Guatemala, Guatemala. (3) Escuela de Nutrición y Dietética. Universidad Santo Tomas, Santiago. Chile.

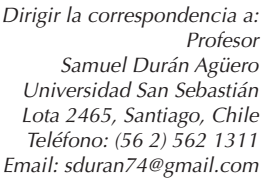

Este trabajo fue recibido el 26 de Marzo de 2013 y aceptado para ser publicado el 1 de Julio de 2013.

están divididos en 2 grandes grupos: Edulcorantes naturales o nutritivos y edulcorantes artificiales o no nutritivos (4).

El primer edulcorante artificial utilizado fue la sacarina la cual fue descubierta por Constantino Fahlberg en 1879 , en Estados Unidos, esta se utilizó principalmente a nivel industrial y como parte de la alimentación de diabéticos. Entre los edulcorantes no nutritivos de mayor consumo podemos nombrar:

Sacarina de sodio: es el edulcorante más antiguo. Esta es una sulfamida, cuyo átomo de hidrogeno es algo ácido y forma sales fácilmente. La sacarina es aproximadamente 300 veces más dulce que el azúcar y no aporta calorías (5), presenta un gusto metálico en altas concentraciones (6).

Ciclamato de sodio: en 1937 fue descubierto en Estados Unidos. Es la sal sódica y cálcica del acido ciclámico presenta una elevada solubilidad en agua. Es 30 a 50 veces más dulce que el azúcar. Es el edulcorante menos intenso por lo que para aumentar su poder endulzante se mezcla con sacarina sódica y así se logra un producto más dulce (5).

Aspartamo: es descubierto en el año 1965 por James Slatter. Es un edulcorante artificial compuesto por un metil ester de un dipéptido formado por el ácido $L$ - aspártico y $\mathrm{L}$ - fenilalanina. Es 180 y 200 veces más dulce que el azúcar y aporta 4 calorías por gramo (5). Debido a que el aspartamo 
contiene fenilalanina, el consumo de éste en las personas que padecen fenilcetonuria está contraindicado, por esta razón los productos que contienen aspartamo deben indicar en la etiqueta "Fenilcetonúricos: contiene fenilalanina". Este producto fue aprobado inicialmente por la FDA en 1980, con algunas restricciones, las cuales fueron anuladas en 1986, quedando el producto, a partir de entonces, libre de restricciones para su registro y venta (7).

Sucralosa: edulcorante artificial descubierto en 1976, compuesto de 1,6 dicloro - 1,6 dideoxy - $\beta$ - D- fructofuranosil - 4 - cloro - 4 deoxy - $\alpha \mathrm{D}$ - galactopiranósido, obtenido por la halogenación selectiva de la molécula de sacarosa. Es entre 500 a 700 veces más dulce que el azúcar, no contiene aporte energético5, es muy soluble en agua y estable bajo condiciones normales de proceso y almacenamiento de bebidas de fantasía. Es pobremente absorbida a través del tracto gastrointestinal (7)

Acesulfamo de potasio: Descubierto en 1967, es un derivado del acetoacético, y es la sal de potasio del 6-metil -1, 2,3 -oxatiazina - 4 (3 H)-1,2, 2- dióxido. Es 160-220 veces más dulce que el azúcar8. Es ligeramente soluble en agua, si bien últimamente se han desarrollado formatos que permiten la preparación de disoluciones concentradas, su estabilidad en estados sólidos es bastante buena siempre que no se sometan temperaturas elevadas (8).

Después de su absorción se excreta sin cambios a través de la orina. No hay evidencias de que se acumule en el organismo. Ya que en algunos ensayos sobre el metabolismo de este compuesto se demostró que no había metabolitos en las excretas de ratas, perros, cerdos y humanos. No se ha demostrado efectos adversos sobre el crecimiento y fertilidad, ni daños hispatológicos, teratogenicidad o cancerogenicidad; sin embargo, se ha sugerido emplearlo con precaución por efectos genotóxicos observados en ratones (9).

Estevia: Estevia rebudiada es una planta selvática subtropical del alto Paraná, nativa del noroeste de la provincia de Misiones, en Paraguay, donde era utilizada por los nativos como medicina curativa, llamada por las tribu de Guaraníes como ka'a he'ê o "yerba dulce". El botánico suizo Moisés Santiago Bertoni fue el primero que la describió, en 1887, detallando su sabor dulce. En 1900, el químico paraguayo Ovidio Rebaudi, logró aislar los principios activos responsables del dulzor (10). Ante la creciente demanda de productos light, la Stevia ha tomado un sitio muy importante en la canasta familiar, se emplea como edulcorante de mesa, en la elaboración de bebidas, dulces, mermeladas, chicles, pastelería, confituras, yogures, entre otros.

Entre sus posibles efectos beneficiosos sobre la salud humana, incluye ser un anti-hipertensivo y anti-hiperglucémico (11).

La planta de estevia produce en las hojas un edulcorante natural, cuyo poder es 300 veces mayor que la sacarosa. No contiene calorías y además, las hojas pueden utilizarse en su estado natural, gracias a su gran poder edulcorante, y sólo son necesarias pequeñas cantidades del producto (12-14).

Neotamo: Su estructura es muy similar a la del aspartamo, la diferencia radica en que el grupo amino del ácido aspártico se cambia por un grupo 3,3 dimetil butilo. Es más estable que el aspartamo. Neotamo tiene un poder endulzante entre $8 \mathrm{mil}$ y 13 mil veces mayor que el azúcar común, por lo que debe ser usado en muy pequeñas cantidades. Fue aprobado por la (FDA) para uso general en julio de 2002, pero todavía no es ampliamente utilizado en productos alimenticios. También está aprobado para su uso en Australia y Nueva Zelanda. Se le asigna el Sistema Internacional de Numeración (SIN) Código de aditivos alimentarios 9615 .

Alitamo fue descubierto por Pfizer Inc. Está formado por dos aminoácidos: ácido L-aspártico y D-alanina, se caracteriza por su elevado poder edulcorante (2.000 veces mayor que el del azúcar). Es soluble en agua y su estabilidad es superior a la del aspartato. Puede potencialmente usarse en prácticamente todas las áreas en que actualmente se usan edulcorantes, por ejemplo: productos de repostería y masas; mezclas secas para bebidas; postres y masas congeladas; goma de mascar y dulces; bebidas calientes y frías ; preparaciones a base de fruta; edulcorantes de mesa; pasta de dientes y enjuagues bucales; productos farmacéuticos; productos lácteos.

Este edulcorante ha sido aprobado para ser usado en una gran variedad de alimentos y bebidas en Australia, Chile, Colombia, Indonesia, Nueva Zelanda, México y la República Popular China. Asimismo, se ha solicitado su aprobación en EEUU, Reino Unido, Canadá, Brasil, varios otros países latinoamericanos y del sudeste de Asia, así como por el Comité Científico de la Alimentación de la Unión Europea (SCF). (Organización de las Naciones Unidas para la Agricultura y la Alimentación/Organización Mundial de la Salud).

La ingesta diaria admisible (IDA) para edulcorantes es de $15 \mathrm{mg}$ kg-1 para acesulfame K, $40 \mathrm{mg}$ kg-1 para aspartamo, 7 mg kg-1 para ciclamato, 5 mg kg-1 para sacarina (15-16), sucralosa $15 \mathrm{mg} \mathrm{kg-1} \mathrm{y} \mathrm{estevia} 4 \mathrm{mg}$ kg-1 (expresado como esteviol) (16).

\section{Ingesta de edulcorantes artificiales y posibles riesgos para la salud}

Durante las últimas décadas la ingesta de edulcorantes y el riesgo de cáncer se ha debatido ampliamente. Un sinnúmero de estudios han sido publicados sobre la ingesta de sacarina en ratas de laboratorio (17-19). Aproximadamente en 20 grupos de estudio, se analizó el efecto de altas dosis de sacarina en una generación de ratas que habían estado expuestos durante 1,5 años. Solo 1 de 20 estudios reportó un significativo aumento de neoplasias (cáncer de vejiga) en los animales alimentados con sacarina al ser comparada con los controles (20). Algunos estudios epidemiológicos anteriores habían encontrado algunos asociación con el riesgo de cáncer de vejiga en seres humanos $(21,22)$. Sin embargo posteriormente se demostró que el metabolismo de la sacarina era específica de la especie, y que la sacarina no daba lugar a litiasis, ya sea del tracto urinario o lesiones epiteliales en los seres humanos (23). Después de la entrada del ciclamato y el aspartamo al mercado de alimentos, enfermedades como el cáncer de vejiga no se podía vincular sólo al consumo de sacarina, porque la mayoría de los consumidores acostumbran a consumir diferentes edulcorantes artificiales (24). Con respecto al consumo de aspartamo un estudio realizado en ratas Sprague-Dawley (900 machos y 900 hembras) fueron tratadas con dosis variables de aspartamo (0 a 100000 ppm) y seguidos hasta su muerte natural, se encontró un exceso aparente de neoplasmas linfáticos sólo en hembras, en el ausencia de una tendencia lineal en el riesgo (25). Un estudio realizado en 1010 casos de diferentes tipos de cáncer confirmado y 2107 controles, no mostró asociación entre el consumo alimentos que contenían edulcorantes (incluyendo el aspartamo) y el riesgo de cáncer (26). Un estudio caso control con 8976 casos con diversos tipos de cáncer y 7028 controles concluyó que no hay asociación entre el consumo de sacarina, aspartame y otros edulcorantes y cáncer (27), otro reciente estudio con 230 casos de cáncer de estómago 
confirmado histológicamente y 547 controles concluyó la ausencia en la asociación en el consumo y el riesgo de neoplasias (28).

En 1970 la FDA prohibió el ciclamato de todos los alimen- tos dietéticos y frutas de los Estados Unidos por sospechar que inducía cáncer en animales de experimentación (29).

El ciclamato se convierte en un metabolito ciclohexilamina, que ha sido informado de su toxicidad (30). Experimentos

TABLA 1

Dulzor, utlización, características generales e IDA de edulcorantes consumidos en Chile.

\begin{tabular}{|c|c|c|}
\hline Edulcorante & Dulzor & $\begin{array}{l}\text { Utilización en la industria } \\
\text { alimentaria }\end{array}$ \\
\hline Sacarina & 300 & $\begin{array}{l}\text { Refrescos, productos horneados, } \\
\text { mermeladas, frutas en conservas, } \\
\text { dulces, aderezos para ensalada, } \\
\text { goma de mascar y edulcorante de } \\
\text { mesa. Además se utiliza en pastas } \\
\text { de dientes, brillo de labios, enjua- } \\
\text { gue bucal, vitaminas y productos } \\
\text { farmacéuticos. }\end{array}$ \\
\hline
\end{tabular}

Ciclamato $\quad 30-50 \quad$ Refrescos, como edulcorante de de sodio

Aspartamo $180-200$

Sucralosa 600

Acesulfa-

$160-220$ mo K
Alimentos y bebidas en general, edulcorante de mesa

Goma de mascar, bebidas carbonatadas, jugos en polvo, yogur y leche, café y té instantáneo, y edulcorante de mesa
Es el edulcorante menos intenso por lo que para aumentar su poder endulzante se mezcla con sacarina sódica y así se logra un producto más dulce.

Los efectos secundarios comunes reportados por el consumo de aspartame incluyen mareos, dolores de cabeza, problemas gastrointestinales, y los cambios del estado de ánimo.

Ingesta de aspartamo durante el embarazo y la lactancia no se ha encontrado para aumentar el riesgo de tumores cerebrales en los niños.

La sucralosa es muy estable y es capaz de retener su dulzor cuando se somete a alta temperatura y acidez. Numerosas pruebas han establecido un perfil de seguridad excelente para la sucralosa, que le permite ser utilizado en todos los grupos de población, incluyendo mujeres embarazadas y lactantes.

El acesulfame $\mathrm{K}$ es estable al calor y se puede utilizar para cocinar y hornear. Por lo general, se combina con el aspartamo o la sucralosa para proporcionar un efecto edulcorante sinérgico. Este tipo de combinaciones no sólo proporcionan un "sabor más parecido al azúcar", pero también disminuyen la cantidad total de edulcorante utilizado.

Estevia aumenta la sensibilidad a la insulina y la tolerancia a la glucosa en los seres humanos.
IDA

$5 \mathrm{mg} / \mathrm{kg}$ de peso corporal por día para los adultos y los niños.

$7 \mathrm{mg} / \mathrm{kg}$ de peso corporal al día.

$50 \mathrm{mg} / \mathrm{kg} \mathrm{de}$ peso corporal, tanto para adultos y niños *40 mg/Kg peso en Chile.

$15 \mathrm{mg} / \mathrm{kg}$ de peso corporal al día.

$15 \mathrm{mg} / \mathrm{kg}$ de peso corporal.

$4 \mathrm{mg} / \mathrm{kg}$ peso corporal (expresados como esteviol). 
realizados con ratas y perros, la ciclohexilamina causa atrofia testicular y alteración de la espermatogénesis $(31,32)$. Takayama y cols. (33) realizaron un estudio de toxicidad a largo plazo con ciclamato en los primates no humanos, veintiún monos fueron alimentados con 100 o $500 \mathrm{mg} / \mathrm{kg}$ de ciclamato/ día por más de 24 años, y se compararon con 16 controles. Los autores concluyeron que no hay evidencia de carcinogenicidad del ciclamato de sodio. No hay estudios descriptivos o de casos y controles de ciclamato en los seres humanos, ya que fue aprobado después de la sacarina, y los productos contienen mezclas de ambos edulcorantes artificiales. Hay que suponer que la mayoría de los consumidores han utilizado tanto la sacarina y el ciclamato desde la introducción de ciclamato.

\section{Edulcorantes, apetito y ganancia de peso}

Recientemente se ha iniciado el debate sobre si el consumo de edulcorantes aumenta el apetito y el peso corporal (34). Sustituir el azúcar por edulcorantes puede ser una estrategia eficaz para el control de peso corporal, aunque los resultados son contradictorios (35-38).

Se ha observado que la ingesta de sacarosa provoca un aumento de la glucosa plasmática y un vaciamiento gástrico más lento al compararlo con sucralosa, quien no provoca cambios en los niveles de glucosa plasmática, al no estimular la liberación de insulina (39), lo mismo sucede con las incretinas cuyos niveles se mantienen estables al consumir edulcorantes, lo que no sucede al consumir sacarosa (40).

Por otro lado se discute que los edulcorantes no poseen un poder saciador como el azúcar, inclusive podrían causar la sensación de hambre estimulando a comer en exceso, además podrían estimular los receptores del gusto, creando adicción al sabor dulce (39).

Estudios que han evaluado el efecto de la estevia sobre el apetito indica que los sujetos que consumieron aspartamo y estevia no compensaron comiendo más en la siguiente comida (almuerzo o cena) y presentaron niveles similares de saciedad en comparación con los sujetos que consumieron sacarosa, adicionalmente estevia redujo los niveles de glucosa plasmática e insulina, lo que sugiere que estevia podría ayudar en la regulación de la glucosa (35).

Varios estudios recientes han documentado las circunstancias bajo las cuales la administración de una variedad de edulcorantes artificiales puede producir respuestas fisiológicas similares a los producidos por la administración de edulcorantes calóricos. Los últimos hallazgos muestran un aumento en la expresión de los transportadores implicados en la absorción intestinal de glucosa (SGLT1) y la inducción de la translocación de los transportadores de glucosa (GLUT2) en el borde en cepillo de la membrana; el aumento dichos cambios pueden facilitar la absorción y el metabolismo de los azúcares ingeridos (41-44). Sin embargo, en el caso de los edulcorantes artificiales, debido a que estos cambios intestinales no están acompañados por la presencia de azúcares ingeridos, como consecuencia se podría aumentar el consumo de energía. Además, estudios recientes han documentado que la sucralosa puede alterar la microflora intestinal y el aumento de peso corporal cuando se administra en las ratas (45).

La evidencia adicional indica que en animales, incluyendo seres humanos, los sabores dulces pueden producir efectos fisiológicos distintos de los producidos por las consecuencias calóricas. Estudios en los seres humanos ha documentado la activación neuronal diferencial en el hipotálamo durante el consumo de edulcorantes calóricos en comparación con los no calóricos, sugiriendo una vez más las diferencias en el consumo de edulcorantes pueden ser detectados (46). En conjunto, estos datos apoyan la hipótesis de que el consumo de los sabores dulces en la ausencia de calorías produce efectos significativamente diferentes en comparación con el consumo de los sabores dulces asociados con las calorías, y con el tiempo estos efectos pueden contribuir a un balance energético positivo y el aumento de aumento de peso corporal.

\section{CONCLUSIONES}

Cada día el consumo de edulcorantes se hace más masivo en nuestra sociedad y están presentes en una gran variedad de alimentos. Los edulcorantes mantienen la palatabilidad de la dieta permitiendo reemplazar el azúcar de muchos alimentos y así reducir el aporte calórico de los mismos. La ingesta es segura, pero es necesario determinar la cantidad de edulcorantes en los alimentos y monitorear el consumo por la población, para determinar si están o no sobrepasando los IDA.

\section{RESUMEN}

Los edulcorantes artificiales son sustancias que no aportan energía y que se agregan a los alimentos para proporcionarles un sabor dulce, incrementando el placer por comer. El objetivo fue revisar la literatura sobre el estado del arte de los edulcorantes artificiales, riesgos de su consumo, posibles efectos en el apetito y ganancia de peso. Desde su introducción, los medios de comunicación han informado sobre los riesgos potenciales de cáncer, lo que ha contribuido a socavar el sentido de la opinión pública de la seguridad. El papel de los edulcorantes en el riesgo de cáncer ha sido ampliamente debatido durante las últimas décadas. Recientes estudios no muestran efectos adversos sobre el consumo de sacarina, aspartame, acesulfamo k y otros edulcorantes, incluyendo datos sobre los cánceres de estómago, páncreas y endometrio. Actualmente muchos edulcorantes artificiales se combinan en los productos actuales, el riesgo cancerígeno de una sustancia única es difícil de evaluar. El consumo de los sabores dulces en la ausencia de calorías produce efectos significativamente diferentes en comparación con el consumo de los sabores dulces asociados con las calorías, y con el tiempo estos efectos pueden contribuir a un balance energético positivo y al aumento de aumento de peso corporal.

Palabras clave: Edulcorantes no nutritivos, apetito, cáncer.

\section{BIBLIOGRAFÍA}

1. Bartoshuk LM. Sweetness: History, Preference, and Genetic Variability. Food Technol, 1991; 45:108-10.

2. Yang $Q$. Gain weight by going diet artificial sweeteners and the neurobiology of sugar cravings, Yale $J$ Biol Med. 2010;83: 101-8.

3. Benton D. ¿Puede ayudar a los edulcorantes artificiales de control de peso corporal y la obesidad prevenir?. Nutr Rev Res. 2005; 18: 63-76.

4. Cagnasso C, López L, Valencia M. Edulcorantes no nutritivos en bebidas sin alcohol: estimación de la ingesta en niños y adolescentes. Arch. Argent Pediatr. 2007;105: 517-21.

5. Polyák E, Gombos K, Hajnal B, Bonyár-Müller K, Szabó S, Gubicskó-Kisbenedek A, Marton K, Ember I. Effects of artificial sweeteners on body weight, food and drink intake. Acta Physiol Hung. 2010; 97:401-7.

6. Mitchell M, Pearson RL. "Saccharin", En: Alternative Sweetener (2 ${ }^{a}$ Edición), O'Brien L, Gelardi RC (Eds.), New York: Marcel Dekker, Inc., 127-56, (1991). 
7. Melis MS, Rocha ST, Augusto ASteviol effect, a glycoside of Stevia rebaudiana, on glucose clearances in rats. Braz $J$ Biol. 2009;69: 371-4.

8. Wells, A.G. The use of intense sweeteners in soft-drinks: En Progress in Sweeteners Grenby TH (ed.), Essex: Elsevier Science Publishers, p 169-214, (1989).

9. Bautista, J., Barboza, E., Gamiño, Z., Alanís, MG. "Alimentos bajos en energía ¿Qué es lo que debemos saber de ellos?". Acta Universitaria.2005;15: 25-33.

10. Abdel-Rahman A, Anyangwe N, Carlacci L, Casper S, Danam $R P$, Enongene $E$, Erives $G$, Fabricant $D$, Gudi $R$, Hilmas CJ, Hines $F$, Howard $P$, Levy $D$, Lin $Y$, Moore RJ, Pfeiler E, Thurmond TS, Turujman S, Walker NJ. The safety and regulation of natural products used as foods and food ingredients. Toxicol Sci. 2011;123: 333-48.

11. Lee CN, Wong KL, Liu JC, Chen YJ, Cheng JT, Chan P. Inhibitory effect of stevioside on calcium influx to produce anti-hypertension. Planta Med. 2001;67:796-9.

12. Brandle JE, Richman A, Swanson AK, Chapman BP. Leaf ESTs from Stevia rebaudiana: a resource for gene discovery in diterpene synthesis. Plant Mol Biol. 2002;50: 613-22.

13. Geuns JM. Stevioside. Phytochem. 2003;64: 913-21.

14. Totté N, Charon L, Rohmer M, Compernolle F, Baboeuf I, Geuns J. Biosynthesis of the diterpenoid steviol, an entkaurene derivative from Stevia rebaudiana Bertoni, via the methylerythritol phosphate pathway. Tetrahedron Letters. 2000;41: 6407-10.

15. Leth $T$, Jenses $U$, Fagt $S$, Andersen R. Estimated intake of intense sweeteners from non-alcoholic beverages in Denmark, 2005. Food Additives Contaminants. 2008; 25: 662-8.

16. Reglamento Sanitario de los Alimentos, articulo 146. Instituto de Nutrición y Tecnología de los Alimentos INTA. Disponible en: http://www.redsalud.gov.cl/portal/url/ page/minsalcl/g_proteccion/g_alimentos/reglamento_sanitario_alimentos.html

17. Weihrauch MR, Diehl V. Artificial sweeteners-do they bear a carcinogenic risk?. Ann Oncol. 2004;15: 1460-5.

18. Hagiwara A, Fukushima S, Kitaori M, Shibata $M$, Ito $N$. Effects of three sweeteners on rat urinary bladder carcinogenesis initiated by $\mathrm{N}$-butyl-N-(4-hydroxybutyl)nitrosamine. Gann. 1984;75:763-8.

19. Olney JW, Farber NB, Spitznagel E, Robins LN. Increasing brain tumor rates: is there a link to aspartame?. I Neuropathol Exp Neurol. 1996;55: 1115-23.

20. Armstrong $B$, Doll $R$. Bladder cancer mortality in diabetics in relation to saccharin consumption and smoking habits. Br J Prev Soc Med 1975;29:73-81.

21. Howe GR, Burch JD, Miller AB, et al Artificial sweeteners and human bladder cancer. Lancet 1977;2:578-81.

22. Capen CC, Dybing E, Rice JM, Wilbourn JD. Lyon, France: IARC; Species differences in thyroid, kidney and urinary bladder carcinogenesis. IARC Scientific Publications No. 147. 1999.

23. Fukushima S, Arai M, Nakanowatari J, Hibino T, Okuda $M$, Ito $N$. Differences in susceptibility to sodium saccharin among various strains of rats and other animal species. Gann; 1983;74: 8-20.

24. Soffritti M, Belpoggi F, Degli Esposti D, et al. First experimental demonstration of the multipotential carcinogenic effects of aspartame administered in the feed to SpragueDawley rats. Environ Health Perspect 2006;114:379-85.

25. Belpoggi $F$, Sofritti M, Padovani M, Degli Esposti D, Lauriola M, Minardi F. Results of long-term cercinogenicity bioassay on Sprague-Dawley rats exposed to aspartame administered in feed. Ann NY Acad Sci, 2006;1076:559-77.

26. Magnuson BA, Burdock GA, Doull J. Aspartame: a safety evaluation based on current use levels, regulations, and toxicological and epidemiological studies. Crit Rev Toxicol. 2007;37: 629-727.

27. Gallus S, Scotti L, Negri E, Talamini R, Franceschi S, Montella M, Giacosa A, Dal Maso L, La Vecchia C. Artificial sweeteners and cancer risk in a network of case-control studies. Ann Oncol. 2007;18:40-4.

28. Bosetti C, Gallus S, Talamini R, Montella M, Franceschi S, Negri $E$, La Vecchia C. Artificial sweeteners and the risk of gastric, pancreatic, and endometrial cancers in Italy. Cancer Epidemiol Biomarkers Prev. 2009 ;18:2235-8.

29. Anonimo. FDA extends ban on cyclamates. Science1970; 169: 962.

30. Renwick AG. The metabolism of intense sweeteners. Xenobiotica1986; 16: 1057-71.

31. Roberts A, Renwick AG. The pharmacokinetics and tissue concentrations of cyclohexylamine in rats and mice. Toxicol Appl Pharmacol. 1989; 98: 230-42.

32. Roberts $A$, Renwick $A G$, Ford $G$ et al. The metabolism and testicular toxicity of cyclohexylamine in rats and mice during chronic dietary administration. Toxicol Appl Pharmacol 1989; 98: 216-29.

33. Takayama S, Renwick AG, Johansson SL et al. Long-term toxicity and carcinogenicity study of cyclamate in nonhuman primates. Toxicol Sci 2000; 53: 33-9.

34. Arcella D, Le Donne C, Piccinelli R, Leclercq C. Dietary estimated intake of intense sweeteners by Italian teenagers. Present levels and projections derived from the INRAN-RM 2001 food survey. Food Chem Toxicol. 2004;42:677-85.

35. Bellisle $F$, Drewnowski A. Intense sweeteners, energy intake and the control of body weight. Eur J Clin Nutr. 2007;61:691-700.

36. Anton SD, Martin CK, Han H, Coulon S, Cefalu WT, Geisselman $P$, Williamson DA. Effects of stevia, aspartame, and sucrose on food intake, saciety, and postprandial glucose and insulin levels. Appetite. 2010;55:37-43.

37. Mattes $R$, Popkin B. Nonnutritive sweetener consumption in humans: effects on appetite and food intake and their putative mechanisms. Am J Clin Nutr. 2009;89:1-14.

38. Blundell JE, Hill AJ. Paradoxical effects of an intense sweetener (aspartame) on appetite. Lancet. 1986; 1: 1092-3.

39. Jing MA, Bellon M, Wishart J, Young R, Blackshaw A, Jones $K$, Horowitz $M$, Rayner $C$. Effect of the artificial sweetener, sucralose, on gastric emptying and incretin hormone release in healthy subjects. Am J of Physiol Gastrointest Liver Physiol.0 2009; 296:735-9.

40. Yukihiro $F$, Rhonda $D$, Speck $M$, Asadi A, King D, Webber $T$, Haneda $M$, Kieffer $T$. Incretin release from gut is acutely enhanced by sugar but not by sweetener in vivo. Am J Physiol Endocrinol Metab. 2009;296: 473-9.

41. Zachary T, Blomgarden MD. Nonnutritive sweeteners, fructose, and other aspects of diet. Diabetes Care. 2011; 34:46-51.

42. Dyer J, Daly K, Salmon KS, Arora DK, Kokrashvili Z, Margolskee RF, Shirazi-Beechey SP. Intestinal glucose sensing and regulation of intestinal glucose absorption. Biochem Soc Trans. 2007;35:1191-4.

43. Egan JM, Margolskee RF. Taste cells of the gut and gastrointestinal hemosensation. Mol Interv. 2008;8:78-81.

44. Margolskee RF, Dyer J, Kokrashvili Z, Salmon KS, Ilegems E, Daly K, Maillet EL, Ninomiya Y, Mosinger B, Shirazi- 
Beechey SP. T1R3 and gustducin in gut sense sugars to regulate expression of $\mathrm{Na+-glucose}$ cotransporter 1. Proc Natl Acad Sci U S A. 2007;104:15075-80.

45. Abou-Donia MB, El-Masry EM, Abdel-Rahman AA, McLendon RE, Schiffman SS. Splenda alters gut microflora and increases intestinal p-glycoprotein and cyto- chrome $p-450$ in male rats. J Toxicol Environ Health $A$. 2008;71:1415-29.

46. Smeets PA, de Graaf C, Stafleu A, van Osch MJ, van der Grond J. Functional magnetic resonance imaging of human hypothalamic responses to sweet taste and calories. Am J Clin Nutr. 2005;82:1011-16. 